\title{
Sustainable Active PET Films by Functionalization With Antimicrobial Bio-Coatings
}

\author{
Annalisa Apicella, Paola Scarfato, Luciano Di Maio and Loredana Incarnato* \\ Department of Industrial Engineering, University of Salerno, Fisciano, Italy
}

The realization of antimicrobial films through the incorporation of active agents into a polymer matrix is a promising alternative to the direct addition of antimicrobials into the food matrix. To this aim, the goal of this work was to develop a sustainable, food packaging solution with antimicrobial effectiveness and high functional performance, based on Ethyl-N $\alpha$-dodecanoyl-L-arginate ( $L A E)$. Active biodegradable coatings, easy soluble to be removed, were realized by spreading a Polylactic acid/LAE coating solution, at different antimicrobial concentration (from 0 to 20\%), on a recyclable Polyethylene-terephthalate substrate. The antimicrobial activity of the multilayer films was tested in vitro against E.Coli CECT 434 strain as pathogenic agent in liquid culture media. Moreover, the LAE chemical interaction with the PLA matrix was investigated,

OPEN ACCESS

Edited by:

Alessandro Pegoretti,

University of Trento, Italy

Reviewed by:

Massimo Messori,

University of Modena and Reggio

Emilia, Italy

Fabrizio Sarasini,

Sapienza University of Rome, Italy

*Correspondence:

Loredana Incarnato

lincarnato@unisa.it

Specialty section:

This article was submitted to Polymeric and Composite Materials,

a section of the journal

Frontiers in Materials

Received: 01 August 2019 Accepted: 18 September 2019 Published: 02 October 2019

Citation:

Apicella A, Scarfato P, Di Maio L and Incarnato $L$ (2019) Sustainable Active PET Films by Functionalization With Antimicrobial Bio-Coatings. Front. Mater. 6:243.

doi: 10.3389/fmats.2019.00243 as well as its effect on the adhesion, wetting, optical and barrier properties of the films. The results pointed out that that the minimum LAE concentration incorporated already guarantees an antimicrobial activity comparable to commercial antimicrobial packaging solutions, and that, among the systems investigated, 10\% LAE is the minimum concentration guaranteeing total inhibition without significantly altering the functionalities of the developed systems.

Keywords: antimicrobial packaging, ethyl-N $\alpha$-dodecanoyl-L-arginate (LAE), polylactic acid (PLA), PET, coating, biodegradable film

\section{INTRODUCTION}

Active packaging solutions have gained increasing attention in the last years from the food industry, since microbial contamination after processing is one of the major causes of foodborne diseases, representing both a public concern and an economic issue (Higueras et al., 2013; Coronel-León et al., 2016). At the same time, consumers demand is increasingly addressed toward the consumption of fresh-like, safe food products, with high organoleptic quality, prolonged shelf-life, and with fewer chemicals addition (Apicella et al., 2019).

The realization of antimicrobial packaging films, through the incorporation of active molecules into the bulk matrix, represents an innovative approach capable to avoid the direct addition, by dipping or spraying, of large antimicrobial amounts onto the food surface, where a large portion of spoilage and contamination occurs. As a matter of fact, the rapid diffusion of the antimicrobial into the food matrix results in an immediate reduction of bacterial populations, but does not allow controlling the metabolism growth of surviving biomass after depletion of antimicrobial residues (Chi-Zhang et al., 2004; Landi et al., 2014). The use of polymers as carriers, instead, allows the gradual delivery of the active agent during the storage and distribution of food packaging, with less antimicrobial concentration, tunable release and tailor-made applications (Lagaron, 2011). 
The main challenges in this field are related to the production of effective packaging systems, with good functional properties, realized through easy-scalable, conventional technologies in use in food packaging industry and, last but not least, with high eco-sustainability (Scarfato et al., 2015a; Apicella et al., 2018a). In fact, the recent concerns toward waste-management issues and shortage of resources, have increasingly shifted the research focus to the development of eco-compatible and sustainable packaging solutions, by means of biomass-derived biodegradable or completely recyclable polymers, or a combination of both (Scarfato et al., 2015b; Zainab and Dong, 2019).

At the same time, the selection of the proper antimicrobial, as well as the choice of its optimal loading, is an important and not an easy task in the design and application of antimicrobial packaging (Becerril et al., 2013; Wicochea-Rodríguez et al., 2019). Some of these substances can modify the organoleptic profile of the packaged foodstuff, or can be used only for a small variety of foods, or can affect the functional properties (i.e., gas permeability, tensile strength, transparency, thermal stability, etc.) of the packaging material, or can have migration limits (European Commission, 2009; Aznar et al., 2013; Muriel-Galet et al., 2014; Paciello et al., 2015; Bugatti et al., 2019).

One of the most innovative and powerful antimicrobial compounds, not showing much of these drawbacks, is Ethyl- $\mathrm{N} \alpha$ dodecanoyl-L-arginate (LAE). LAE is a derivative of lauric acid, L-arginine and ethanol, which showed an extensive spectrum of antimicrobial activity against Gram positive and Gram-negative bacteria, as well as yeasts and molds (Infante et al., 1997; Bakal and Diaz, 2005; Pezo et al., 2012). It interacts, as a cationic surfactant, on the cytoplasmic membrane of microorganisms increasing the cell permeability without causing lysis (Nerin et al., 2016). Among the major positive features, LAE has been classified as Generally Recognized as Safe (GRAS) and food preservative by the Food and Drug Administration (FDA), is chemically stable, has low cost, and does not provide any taste or odor, in compliance with European Regulation on active food packaging materials (Otero et al., 2014).

Previous published researches already addressed the realization of packaging films incorporated with LAE, realized by solvent casting technique (Muriel-Galet et al., 2012; Rubilar et al., 2016; Moreno et al., 2017; Haghighi et al., 2019). However, to the best of our knowledge, limited information is available concerning the development of sustainable packaging solutions based on PET films, functionalized with LAE-activated bio-coatings, and produced with conventional techniques easy-scalable at industrial level.

On the basis of the aforementioned considerations, the goal of this study was to develop antimicrobial, multifunctional, and sustainable food packaging films, based on LAE, by means of coating technology. The multilayer films were realized by spreading an amorphous PLA coating layer (C), incorporated at different concentrations of LAE, on a Bi-oriented PET substrate (S). PET was selected as web layer thanks to its excellent functional properties, as oxygen and water vapor barrier, high tensile strength, and thanks to its complete recyclability (Di Maio et al., 2017). Amorphous PLA coating already demonstrated to provide sealant layer to polyester films (Barbaro et al., 2015), in addition to its good optical properties, good processability, and environmental benefits (Scarfato et al., 2017). In addition, the biodegradable layer is easy to be removed by non-toxic solvents, ensuring the complete recovery of the recyclable substrate (Bugnicourt et al., 2013), and the coating technology ensures to avoid thermal stresses to heat sensitive active compounds.

The antimicrobial effectiveness of the active films was assessed by means of microbiological tests, using E. Coli CECT 434 strain as pathogenic agent. Moreover, the produced systems were characterized in order to ascertain the effect of the active phase on the chemical, morphological, and functional properties of the polymeric films.

\section{MATERIALS AND METHODS}

\section{Materials}

Commercial biaxially oriented poly(ethylene terephthalate) (BOPET) film (Nuroll S.p.a, Italy), with $23 \mu \mathrm{m}$ thickness and corona treated surface, was used as substrate (S). PLA4060 (Natureworks, Minnetonka, USA), characterized by a D-lactide content of $12 \mathrm{wt} \%$, was used for the coating layer (C). Ethyl-N $\alpha$ dodecanoyl-L-arginate (LAE) was provided by Vedeqsa Grupo LAMIRSA (Terrassa, Barcelona, Spain). All the solvents used were analytical grade.

\section{Preparation of the Active Systems}

Antimicrobial coated films were realized according to the method explained by Barbaro et al. (2015), with some modifications. The PLA coating solution was prepared by dissolving the polymer in acetone (mass ratio 20:80) and subsequently adding LAE at different percentages $(0,5,10$, and $20 \% \mathrm{w} / \mathrm{wt})$. The casting mixture was spread on the BOPET substrate by means of a K Hand Coater (RK, Printocoat Instruments Ltd., Litlington, UK), equipped with stainless steel closed wound rod, with wire diameter equal to $0.64 \mathrm{~mm}$, yielding final coatings with an average thickness of the coating layer comprised between 7 and 10 microns.

Table 1 resumes the list of the prepared films (named SC, SC5, SC10, and SC20). The PET substrate (S) was also used as comparison.

\section{Characterization Methods ATR-FTIR Analyses}

Fourier Transform Infrared spectra of the films were collected by a Thermo Scientific Nicolet 600 FT-IR, equipped with a

TABLE 1 | List of the prepared systems, at different percentages of active phase.

\begin{tabular}{lccc}
\hline $\begin{array}{l}\text { Sample } \\
\text { film }\end{array}$ & $\begin{array}{c}\text { LAE } \\
\text { concentration } \\
(\mathbf{w t} \%)\end{array}$ & $\begin{array}{c}\text { Thickness of } \\
\text { the coating } \\
\text { layer }(\boldsymbol{\mu} \mathbf{m})\end{array}$ & $\begin{array}{c}\text { Total } \\
\text { thickness } \\
(\boldsymbol{\mu} \mathbf{m})\end{array}$ \\
\hline S & 0 & 0 & 23 \\
SC & 0 & $7 \pm 0.5$ & $30 \pm 0.5$ \\
SC5 & 5 & $7 \pm 0.9$ & $30 \pm 0.9$ \\
SC10 & 10 & $8 \pm 1.0$ & $31 \pm 1.0$ \\
SC20 & 20 & $10 \pm 1.2$ & $34 \pm 1.2$
\end{tabular}


Smart Performer accessory for attenuated total-reflection (ATR) measurement using a $\mathrm{ZnSe}$ crystal. The operating spectral range was set at $650-4,000 \mathrm{~cm}^{-1}$, with a resolution of $4 \mathrm{~cm}^{-1}$ and 64 scans per sample. Normalization and peak integration was performed using Omnic software.

\section{Field Emission Scanning Electron Microscopy (FESEM) Analyses}

Field Emission Scanning Electron Microscopy (SEM) analyses were conducted on the film sections, which were cut cryofracturing them in liquid nitrogen normally to the extrusion direction, sputter coated with gold (Agar Auto Sputter Coater mod. 108A, Stansted, UK) at $30 \mathrm{~mA}$ for $160 \mathrm{~s}$, and analyzed using a field emission scanning electron microscope (mod. LEO 1525, Carl Zeiss SMT AG, Oberkochen, Germany).

\section{Delamination Tests}

In order to evaluate the adhesion strength of the PLA coatings, delamination tests were carried out by SANS dynamometer (mod. CMT 4000 by MTS, China), equipped with a $10 \mathrm{~N}$ load cell, according with the standards ASTM F88-00 and ASTM 2029. In particular, the coated films were cut in strips of $200 \times 25$ $\mathrm{mm}^{2}$ area, sealed with a Brugger HSG-C (Germany) heat sealing machine at $120^{\circ} \mathrm{C}$ for $1 \mathrm{~s}$, by applying a force of $690 \mathrm{~N}$, and stored for $48 \mathrm{~h}$ in environmental condition prior to analysis. Then, the bonding strength was evaluated in tensile mode at 250 $\mathrm{mm} / \mathrm{min}$ until delamination failure of the seal. The maximum load reported is considered as the maximum bond strength of the coating on a specific substrate (expressed as N/25 mm). For each sample type, at least 10 measurements were performed to assess the reproducibility of the results.

\section{Static Water Contact Angle and Surface Tension Characterization}

Static contact angle measurements were performed with a First Ten Angstrom Analyzer System 32.0 mod. FTA 1000 (First Ten Angstroms, Inc., Portsmouth, VA, USA), according to the standard test method ASTM D5946. The drop volume was taken within the range where the contact angle did not change with the variation of the volume $(2 \pm 0.5 \mu \mathrm{L})$. Each reported value of the $\theta$ angle is the average of at least 10 replicate measurements. The dispersion $\left(\gamma_{\mathrm{s}}^{\mathrm{d}}\right)$ and polar $\left(\gamma_{\mathrm{s}}^{\mathrm{p}}\right)$ components of the surface energy (SE) for all the samples were calculated according to the Owens-Wendt geometric mean equation (Owens and Wendt, 1969), using distilled water and ethylene glycol as testing liquids. The SE components $(\mathrm{mN} / \mathrm{m})$ for water are: $\gamma=72.1, \gamma^{\mathrm{d}}=19.9$, $\gamma^{\mathrm{p}}=52.2$, and for ethylene glycol are: $\gamma=48, \gamma^{\mathrm{d}}=29, \gamma^{\mathrm{p}}=19$ (here $\gamma$ is the total SE, and $\gamma^{\mathrm{d}}$ and $\gamma^{\mathrm{p}}$ are the dispersion and polar components, respectively) (Zonder et al., 2014).

\section{Antimicrobial Activity Assays}

The antimicrobial effectiveness of the films was tested against the Gram-negative bacteria E. Coli CECT 434 (ATCC 25922), selected because of its relevance in food industry. Stock culture was stored at $-18^{\circ} \mathrm{C}$ in Nutrient Broth (Sigma-Aldrich, Missouri, USA) with $40 \%$ Glycerol. Subcultures were grown overnight in
Tryptic Soy Broth (TSB, Sigma-Aldrich) plus 0.6\% Yeast Extract (YE, Sigma-Aldrich) at $37^{\circ} \mathrm{C}$ prior to each experiment.

The minimum inhibitory concentration (MIC) and the minimum bactericidal concentration (MBC) of LAE against the selected strain was determined in TSBYE. $1.2 \mathrm{~g}$ of LAE was diluted in $100 \mathrm{~mL}$ of Milli-Q water to obtain a concentration of $12,000 \mathrm{ppm}$, and then serial dilutions between 80 and $4 \mu \mathrm{g} / \mathrm{mL}$ were made up in sterile TSBYE to study MIC and MBC. Hundred microliter of $\sim 10^{4} \mathrm{CFU} / \mathrm{mL}$ of microorganism in exponential phase was inoculated in each test tube. The tubes were incubated at $37^{\circ} \mathrm{C}$ overnight, then, $100 \mu \mathrm{L}$ from each tube was plated and incubated at $37^{\circ} \mathrm{C}$ overnight (M100-S18, 2008). MIC was reported as the lowest antimicrobial concentration that inhibited the growth of the pathogen microorganism, while the $\mathrm{MBC}$ was defined as the lowest concentration at which no colonies growth was observed in the medium (TSBYE), and they were not culturable after plating onto Tryptic Soy Agar (TSA, SigmaAldrich) (Muriel-Galet et al., 2012; Higueras et al., 2013).

In vitro microbial tests on the films were performed by cutting $2.5 \times 2.5 \mathrm{~cm}^{2}$ of multilayer film samples at different LAE concentration $(0,5,10$, and $20 \%)$, sterilizing them by UV lamp on both sides, and placing them in a sterile tube containing $5 \mathrm{ml}$ of TSBYE medium. Aliquots containing $100 \mu \mathrm{L}$ of $\sim 10^{4} \mathrm{CFU} / \mathrm{mL}$ of microorganism in exponential phase were inoculated in each test tube, and incubated at $37^{\circ} \mathrm{C}$ and $300 \mathrm{rpm}$ overnight. Depending on the turbidity of the tubes, serial dilutions with physiological saline were made and plated in Petri dishes with $20 \mathrm{~mL}$ TSA culture medium. Colonies visible to naked eye were counted after incubation at $37^{\circ} \mathrm{C}$ overnight (Paciello et al., 2013). The negative controls (i.e., films in liquid medium without $E$. Coli) were also prepared. Counts were performed in triplicate.

\section{Water Vapor Permeability Tests}

Water vapor permeability was measured by M7002 Water Vapor Permeation Analyzer (Systech Instruments Ltd, Oxfordshire, UK) according to the standard ASTM F 1249. Films were tested at $23^{\circ} \mathrm{C}$ and $50 \%$ R.H., and the results, performed in triplicate, were expressed as PWV $\left(\mathrm{g} \mathrm{m} /\left(\mathrm{m}^{2} \mathrm{~Pa} \mathrm{~s}\right)\right)$, calculated as the following equation (Li et al., 2015):

$$
P_{W V}=\frac{W V T R \times L}{\Delta P}
$$

where WVTR is the water vapor transmission rate $\left(\mathrm{g} / \mathrm{m}^{2} \mathrm{~s}\right)$ measured through the film, $\mathrm{L}$ is the average film thickness $(\mathrm{m})$, and $\Delta \mathrm{P}$ is the partial water vapor pressure difference $(\mathrm{Pa})$ across the two sides of the film.

\section{Optical Analyses}

The optical properties of the films were evaluated by measuring the UV-Visible transmittance of the films from 200 to $800 \mathrm{~nm}$ with Perkin Elmer UV-Visible Spectrophotometer Lambda 800. The transparency of the films was evaluated by measuring the Transmittance \% of visible light at $550 \mathrm{~nm}$, according to the ASTM D1746-03. 


\section{RESULTS AND DISCUSSION ATR-FTIR Analyses}

Possible changes in intra- and intermolecular interactions due LAE addition into the PLA matrix were explored by Fourier transform infrared spectroscopy (FT-IR), which is sensitive to the structural conformation and local molecular motions. Due to the limited penetration depth of infrared radiation into the sample in the ATR-FT-IR measurement geometry, the spectra were collected on the thin PLA coating layers (namely C, C5, C10, and C20), at different percentages of antimicrobial, and compared to the spectrum of LAE, which is shown in Figure 1A. The absorption band at $3,320 \mathrm{~cm}^{-1}$ corresponds mainly to the $v(\mathrm{~N}-\mathrm{H})$ stretching vibration of hydrogen bonded $\mathrm{N}-\mathrm{H}$ functionalities. The double peaks at 2,927 and $2,850 \mathrm{~cm}^{-1}$ can be assigned to antisymmetric and symmetric stretching vibrations of $\mathrm{CH}_{3}$ and $\mathrm{CH}_{2}$ functionalities, $v_{\text {as }}\left(\mathrm{CH}_{3} / \mathrm{CH}_{2}\right)$ and $\nu_{\mathrm{s}}\left(\mathrm{CH}_{3} / \mathrm{CH}_{2}\right)$, respectively. The small peak around $1,740 \mathrm{~cm}^{-1}$ suggest the presence of carbonyl groups, the one at 1,560 $\mathrm{cm}^{-1}$ corresponds to the $\delta(\mathrm{N}-\mathrm{H})$ bending vibrations (amideII) combined with $v(\mathrm{C}-\mathrm{N})$ stretching, the peak at $1,655 \mathrm{~cm}^{-1}$ is due to the $v(\mathrm{C}=\mathrm{O})$ stretching vibration (amide-I), while the band situated around $1,027 \mathrm{~cm}^{-1}$ can be assigned to a $v(\mathrm{C}-\mathrm{O})$ stretching vibration. Similar outcomes shown by other authors (Haghighi et al., 2019).

As it is possible to observe from Figures 1B-D, the PLA characteristic absorption peaks remains essentially unaltered, and the LAE characteristic absorption peaks are observable only by increasing the antimicrobial content in the coating layers. In particular, for the samples at 10 and $20 \% \mathrm{LAE}$ (C10 and C20, respectively), the absorption bands at 2,927, 2,850, 1,655, and 1,027 $\mathrm{cm}^{-1}$ are detectable. The absence of changes in the intensities and positions of the major PLA and LAE bands suggests the realization of a physical mixture, with no chemical interaction between the two phases. Similar results were also reported by other authors (Rubilar et al., 2016; Gaikwad et al., 2017).

\section{FESEM Analyses}

As it is known, the microstructural properties of composite films depend on the compatibility between all the film components, affecting the final physical, mechanical, barrier, and optical properties (Attaran et al., 2015). To this aim, the distribution of the active phase inside the PLA matrix and the quality of the interlayer adhesion were investigated through SEM analyses. Figures 2A,B show the cross-section micrographs of SC and SC10 films, respectively, taken as an example. Images analyses display the PLA coating layer, in which few small voids are recognizable due to solvent evaporation, over the BOPET substrate, characterized by an oriented morphology. The absence of visible LAE powder domains inside the coating layer highlights the good dispersion and homogeneous distribution of the antimicrobial into the polymer matrix. Moreover, the absence of voids in the cross sectional area of the investigated films pointed out the good adhesion of the coating layers on the substrate.

\section{Evaluation of Adhesion Strength and Surface Wettability}

To determine the adhesion strength, delamination tests were performed as described in the methods section. The delamination occurred by separating the coating, thermally sealed at the defined temperature, by means of a dynamometer. The bonding strength, reported in Table 2, represents the force required to delaminate the PLA coating from the web.

The results show that the adhesion strength of the PLA coating layers was not significantly affected by the antimicrobial addition up to LAE concentrations equal to $10 \%$ (SC10 sample), whereas a decrease in the bonding strength for the SC20 film, equal to 2.56 $\pm 0.35 \mathrm{~N} / 25 \mathrm{~mm}$, was observed.

The adhesion strength strictly depends on the amount of polar and dispersion bonds between the substrate film and the coating (Lindner et al., 2017).

To this aim, the polar $\left(\gamma_{\mathrm{s}}^{\mathrm{p}}\right)$ and dispersion $\left(\gamma_{\mathrm{s}}^{\mathrm{d}}\right)$ components of the surface energy were calculated from water and ethylene glycol static contact angle measurements, as described in Par. 2.3.4, and displayed in Table 2.

The reported surface energy values show that the adhesion force is optimal when the $\gamma_{\mathrm{s}}^{\mathrm{p}}$ and $\gamma_{\mathrm{s}}^{\mathrm{d}}$ values are of the same order of magnitude, and maximum when their ratio is close to unity. On the other hand, when the difference between the $\gamma_{s}^{\mathrm{p}}$ and $\gamma_{s}^{\mathrm{d}}$ values is more pronounced, as in the case of SC20 sample, where it is of one order of magnitude, a significant drop in bonding strength is observable, which however remains acceptable for the film application in food packaging.

\section{Antimicrobial Activity of the Active Bio-Coated Films With LAE}

The antimicrobial activity of the multilayer films against $E$. Coli growth was then determined by the liquid medium method described in the experimental section.

A preliminary study, previously carried out on only the PLA coating layers, showed the antimicrobial effectiveness of the coatings in releasing the active agent and inhibiting the proliferation of microorganisms, with a total inhibition at the LAE concentration of $20 \%$ (Apicella et al., 2018b).

However, in the real packaging applications, the release does not occur symmetrically on both sides of the coating, and the different layout of systems can determine differences in the mass transport of the active agent. Therefore, it is necessary to study in depth the antimicrobial efficacy of the active multilayer films in real geometry and application conditions.

In first analysis, the MIC and the MBC for LAE were evaluated in the test conditions described in the methods section. The corresponding values are reported in Table 3 , and are equal to 51 and $63 \mathrm{ppm}$, respectively. These values, different from previous results reported in the literature, highlight the influence of medium composition, of the method used (in terms of incubation time, inoculum concentration, and temperature), as well as of the strain used, in the quantification of the activity of the antimicrobial agent (Muriel-Galet et al., 2012).

Afterwards, the microbiological tests were conducted on the SC samples, at different LAE concentrations $(0,5,10$, and 20\%) 


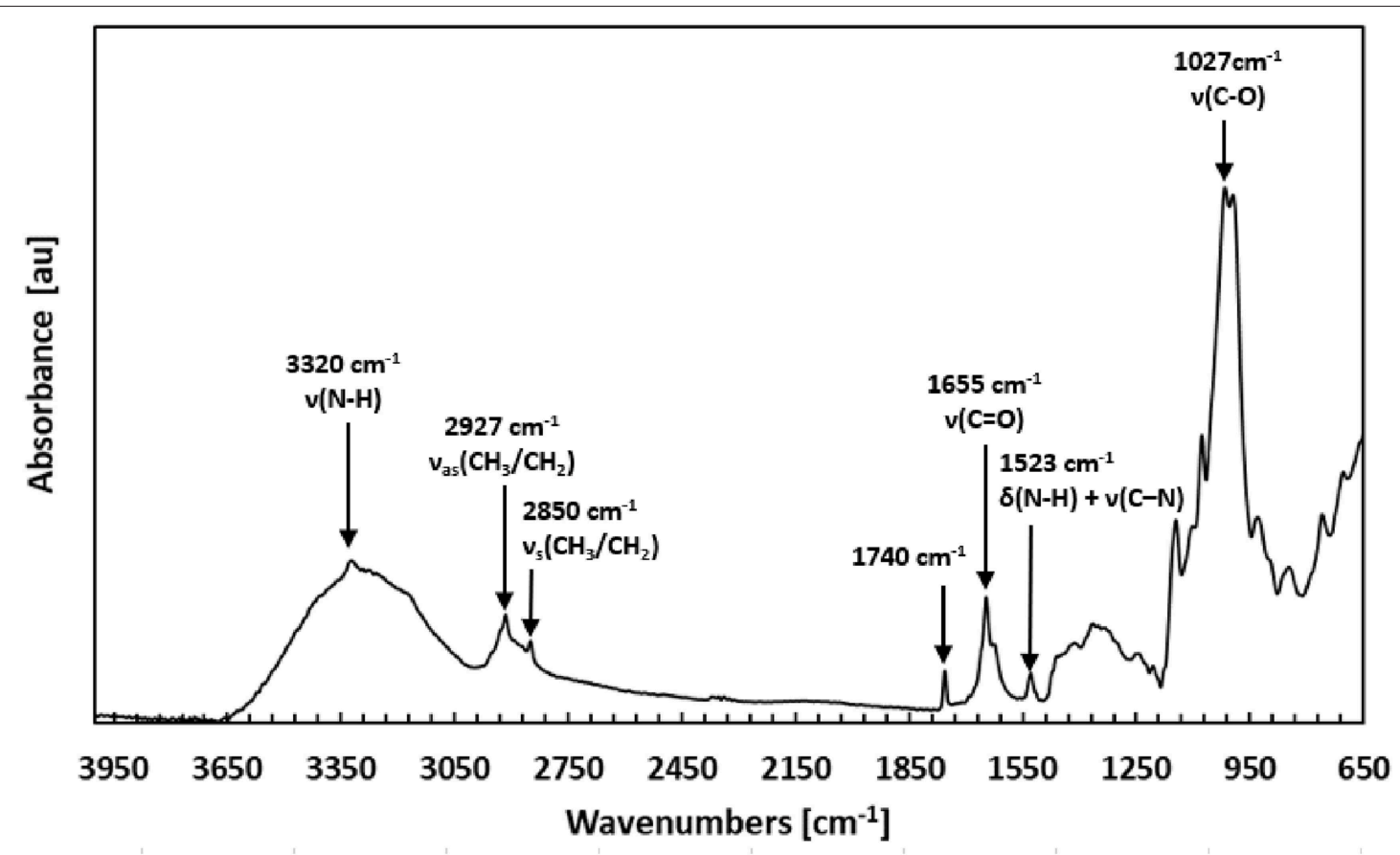

A

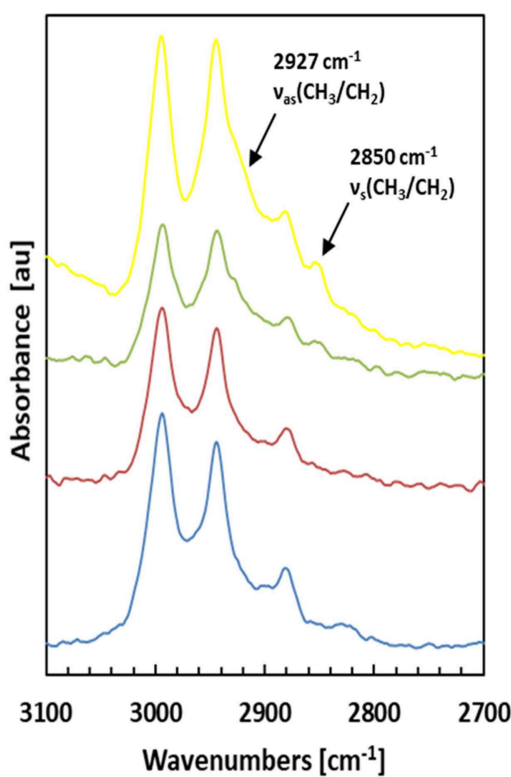

B

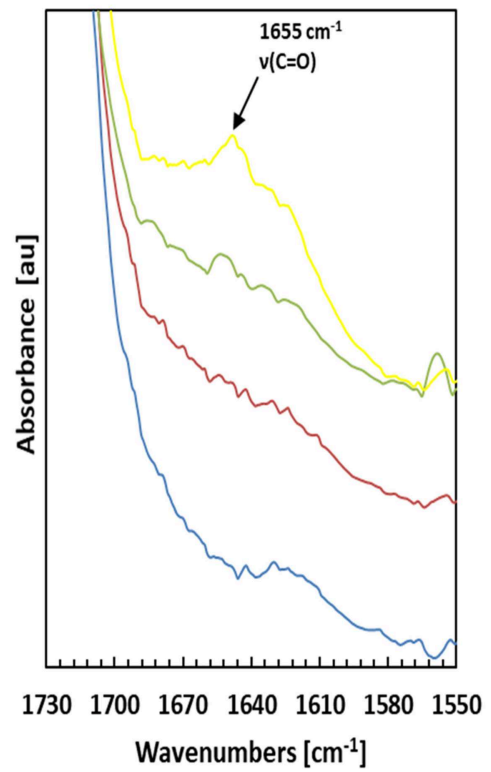

C

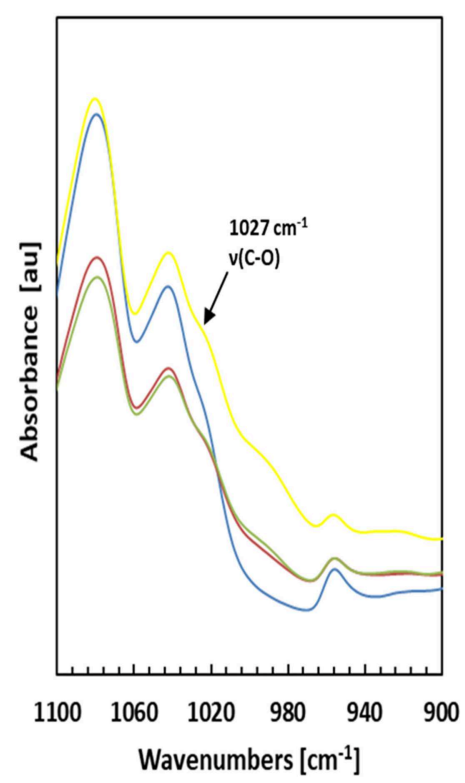

D

\section{$\mathrm{C}-\mathrm{C} 5-\mathrm{C} 10-\mathrm{C} 20$}

FIGURE 1 | ATR-FTIR spectra of LAE (A) and of the thin PLA coating layers C, C5, C10, and C20, loaded at 0, 5, 10, and 20\% LAE, respectively (B-D).

and with exposed surface area equal to $6.25 \mathrm{~cm}^{2}$, according to the conditions described in the experimental section.

The results are reported in Table 3, and are expressed as logarithm of colony forming units $(\log (\mathrm{CFU}))$ and $\log$ reduction value (LRV). Data regarding the control sample are also reported as reference. For further comprehension, Figure 3 shows the pictures of the tested tubes after incubation at $37^{\circ} \mathrm{C}$ overnight, in comparison with the control sample. 

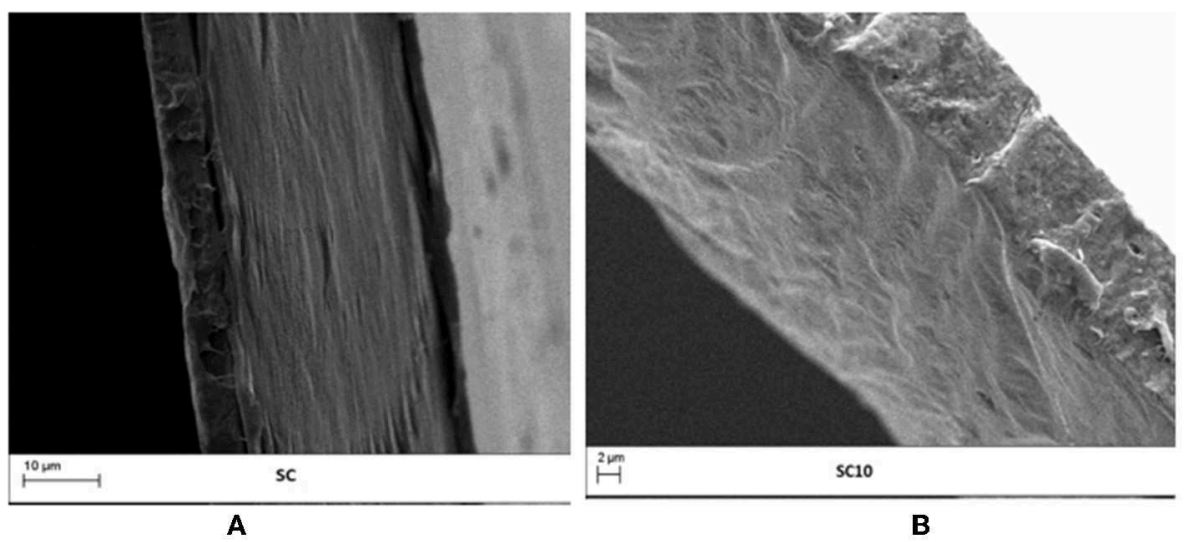

FIGURE 2 | Cross-sectional SEM micrographs of: (A) SC and (B) SC10 films.

TABLE 2 | Bonding strenght (N/25 mm), static water (CAw), and ethylene glycol $\left(\mathrm{CA}_{E G}\right)$ contact angles, and dispersion $\left(\gamma_{S}^{\mathrm{d}}\right)$ and polar $\left(\gamma_{S}^{\mathrm{p}}\right)$ components of the surface energy for the neat PET substrate film (S) and for the SC coated films at 0 , 5, 10, and 20\% LAE concentration.

\begin{tabular}{lccccc}
\hline $\begin{array}{l}\text { Sample } \\
\text { film }\end{array}$ & $\begin{array}{c}\text { Bonding } \\
\text { strenght } \\
(\mathbf{N} / 25 \mathbf{~ m m})\end{array}$ & $\mathbf{C A w}\left(^{\circ}\right)$ & $\mathbf{C A}_{\mathbf{E G}}\left(^{\circ}\right)$ & $\gamma_{\mathbf{S}}^{\mathbf{d}}$ & $\gamma_{\mathbf{S}}^{\mathbf{p}}$ \\
\hline S & Not sealable & $65.3 \pm 2.8$ & $39.5 \pm 0.9$ & 18.8 & 19.3 \\
SC & $3.20 \pm 0.4$ & $65.8 \pm 1.0$ & $54.3 \pm 1.3$ & 7.4 & 28.6 \\
SC5 & $3.12 \pm 0.3$ & $55.9 \pm 1.1$ & $43.1 \pm 1.2$ & 7.9 & 36.5 \\
SC10 & $3.10 \pm 0.8$ & $54.2 \pm 1.3$ & $41.3 \pm 1.1$ & 7.9 & 38.1 \\
SC20 & $2.56 \pm 0.3$ & $46.4 \pm 2.2$ & $40.5 \pm 1.3$ & 4.2 & 51.4 \\
\hline
\end{tabular}

TABLE 3 | Minimum inhibitory concentration (MIC) and minimum bactericidal concentration (MBC) of LAE against $E$. Coli, and antimicrobial activity for the SC coated films at $0,5,10$, and 20\% LAE concentration., expressed as logarithm of colony forming units (Log(CFU)) and log reduction value (LRV).

\begin{tabular}{lcccc}
\hline Sample & \multicolumn{4}{c}{ Escherichia Coli CECT 434 } \\
\cline { 2 - 5 } & $\begin{array}{c}\text { MIC } \\
\text { (ppm) }\end{array}$ & $\begin{array}{c}\text { MBC } \\
\text { (ppm) }\end{array}$ & Log(CFU) & LRV \\
\hline LAE & 51 & 63 & - & - \\
Control & - & - & $10.96 \pm 0.69$ & - \\
SC & - & - & $10.88 \pm 1.22$ & 0 \\
SC5 & - & - & $5.79 \pm 0.60$ & 5.17 \\
SC10 & - & - & \multicolumn{2}{r}{ Total inhibition } \\
SC20 & - & - & \multicolumn{2}{r}{ Total inhibition } \\
\hline
\end{tabular}

As expected, the unloaded SC sample was not effective in inhibiting the microbial growth, with an LRV value equal to 0 and a consistent turbidity in all the tubes containing the film samples (Figure 3A). On the other hand, the LAE addition caused a consistent ( $>5 \mathrm{log}$ ) reduction of the microorganism viable counts even at the lowest percentage analyzed. In fact, the multilayer film loaded at 5\% LAE (SC5) caused a growth reduction of $5.17 \log$ against $E$. Coli, while LAE concentration equal to $10 \%$, or higher, produced total inhibition against the microorganism tested. What is more, the pictures comparison in Figures 3B-D showed a progressive increase in the optical clarity when compared to the control sample, and the disappearance of the bottom cellular deposit at LAE 10 and 20\% (SC10 and SC20, respectively). It is worth to point out that the LRV value obtained even at the lowest concentration of LAE, indicates at least a partial deliver of the agent sufficient to achieve an antimicrobial effect comparable with or higher than currently commercially available antimicrobial films and coatings for foods and pharmaceuticals (Molling et al., 2014), while the 10\% LAE concentration is the minimum guaranteeing total inhibition of microbial growth among the investigated systems.

These promising results highlight that the developed packaging solutions are efficient even at the minimum concentration of active agent investigated, and pave the way to more in depth studies on the release kinetics, aimed at optimizing the films in terms of antimicrobial concentration and coating thickness.

\section{Water Vapor Permeability (WVP) Measurements}

In order to better investigate the effects of the systems composition on the barrier performance of the samples, water vapor permeability tests were carried out, and outcomes are reported in Table 4. All the coated films (SC), with respect to the PET substrate (S), show an increase in the water vapor permeability values. However, this is mainly due to the normalization over the total thickness of the films, in which the amorphous PLA layer does not offer any resistance to the transport of water molecules (Robertson, 2013; Halász et al., 2015). A slight further increase in the water vapor permeability is observable at the highest LAE content, i.e., in the SC20 sample.

\section{Optical Analyses}

In packaging applications, the transmission of visible and ultraviolet light are important parameters to preserve and protect 


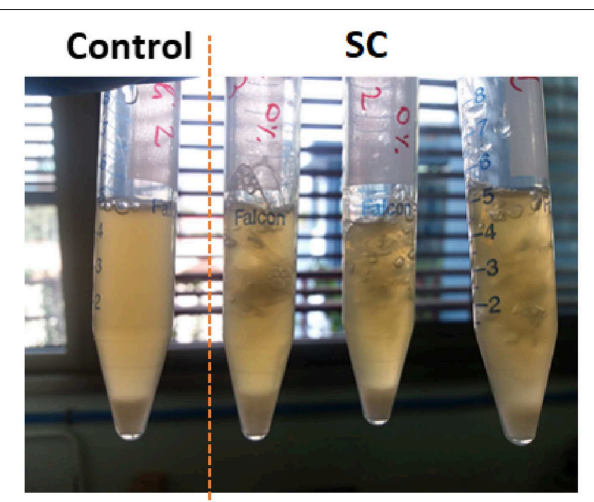

A

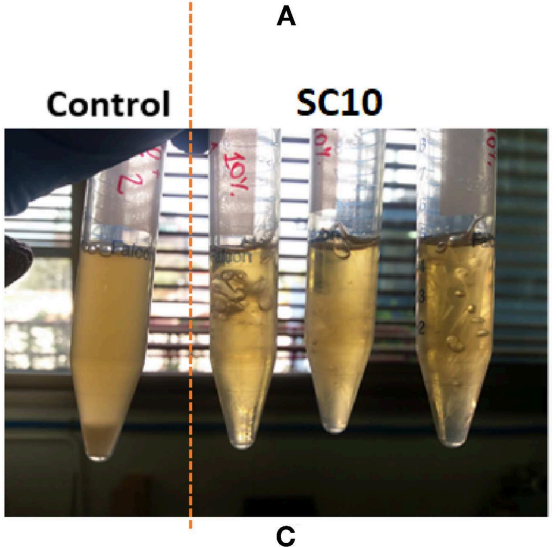

C

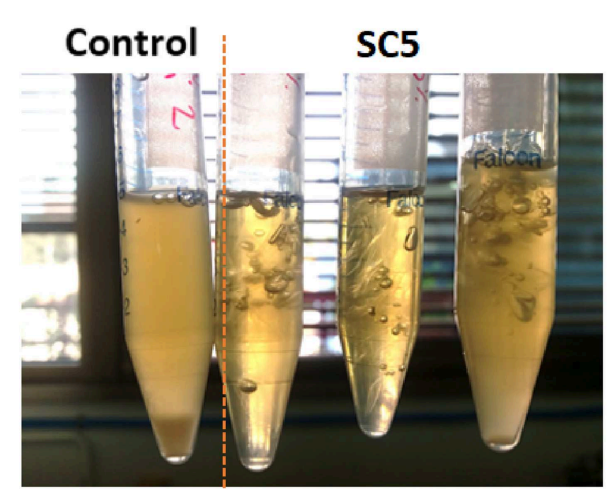

B

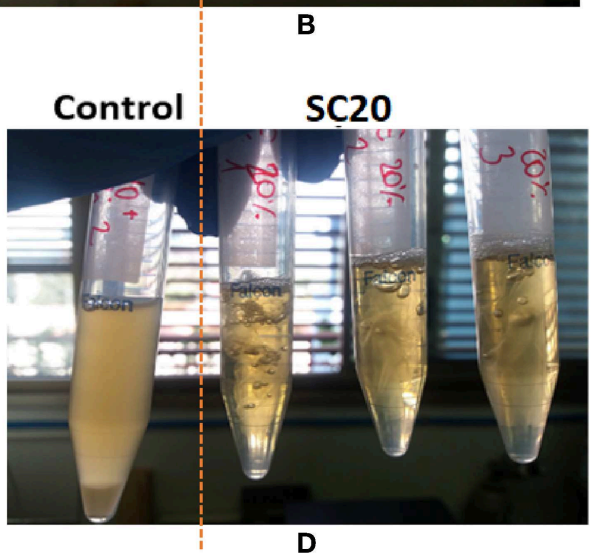

FIGURE 3 | Images of the tested safe-lock tubes inoculated with E. Coli and containing the SC coated films produced at different concentration of active agent: 0\% LAE (A), 5\% LAE (B), 10\% LAE (C), and 20\% LAE (D), after incubation at $37^{\circ} \mathrm{C}$ overnight, in comparison with the control sample.

TABLE 4 | Water vapor permeability (PWV) and UV-Vis transmittance at $550 \mathrm{~nm}$ $\left(T_{550} \%\right)$ for the neat PET substrate film (S) and for the SC coated films at 0, 5, 10, and $20 \%$ LAE concentration.

\begin{tabular}{lcc}
\hline Sample film & $\mathbf{P}_{\mathbf{W v}}{ }^{*} \mathbf{1 0}^{\mathbf{1 2}} \mathbf{( \mathbf { g ~ m } / ( \mathbf { m } ^ { \mathbf { 2 } } \mathbf { P a ~ s } )}$ & $\mathbf{T}_{\mathbf{5 5 0}} \mathbf{( \% )}$ \\
\hline S & $0.74 \pm 0.01$ & 84.3 \\
SC & $1.11 \pm 0.04$ & 84.3 \\
SC5 & $1.12 \pm 0.02$ & 73.2 \\
SC10 & $1.14 \pm 0.02$ & 69.1 \\
SC20 & $1.25 \pm 0.12$ & 60.1 \\
\hline
\end{tabular}

food products until they reach the consumer, as well as to get an attractive transparent package. To evaluate the transparency of samples, UV-Vis measurements were carried out. PET and PLA are known to have excellent optical properties, in this sense the LAE effect on transparency was investigated. In UV-Vis spectra reported in Figure 4, the transmittance percentage T\% is reported as a function of wavelength for the neat substrate (S) the coated SC films at different content of the active phase. The transparency of the multilayer films, defined as the transparency of visible light in short range of $540-560 \mathrm{~nm}$, was therefore evaluated measuring the transmission at $550 \mathrm{~nm}\left(\mathrm{~T}_{550} \%\right)$, and the values for the investigated samples are reported in Table 4.

As observable from the comparison among the S and SC samples, the PLA coating did not affect the transparency of the

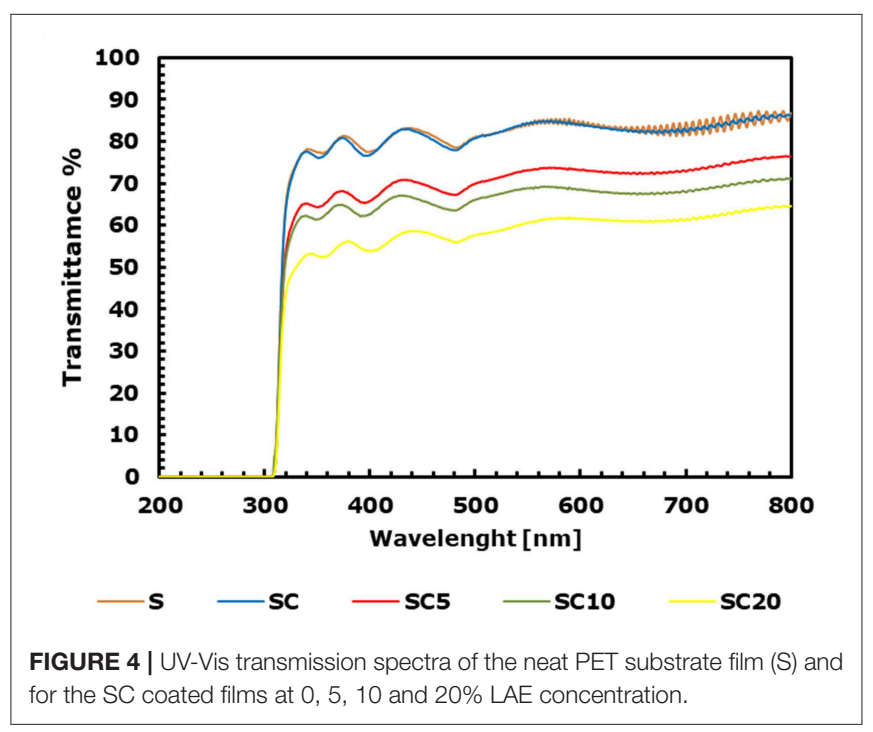

PET substrate, which remains the highest, with a $\mathrm{T}_{550} \%$ value equal to $84.3 \%$ in both films. The further LAE addition to the polymer matrix determines a slight decrease in the transmittance at $550 \mathrm{~nm}$, which becomes more significant at LAE concentration equal to $20 \%$ ( $\mathrm{T}_{550} \%$ of SC20 equal to $60.1 \%$ ). In this latter case, 


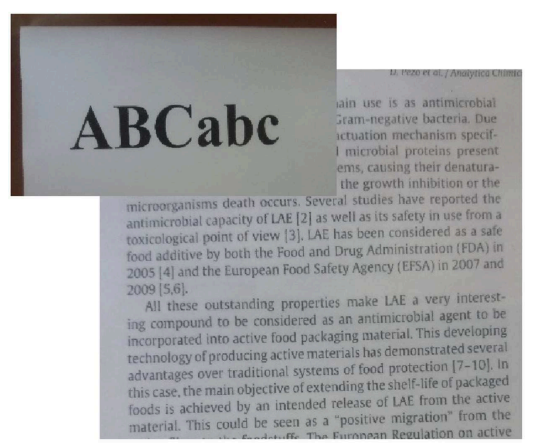

A

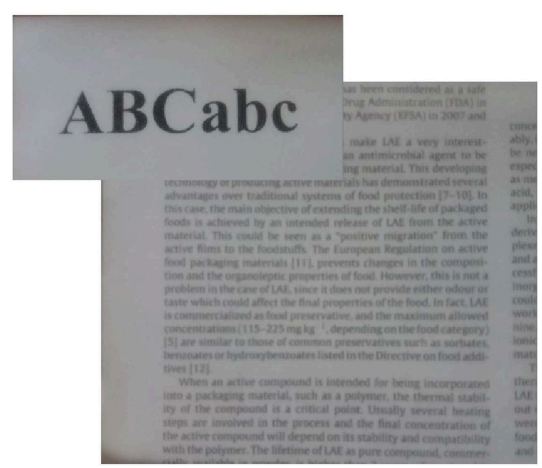

C

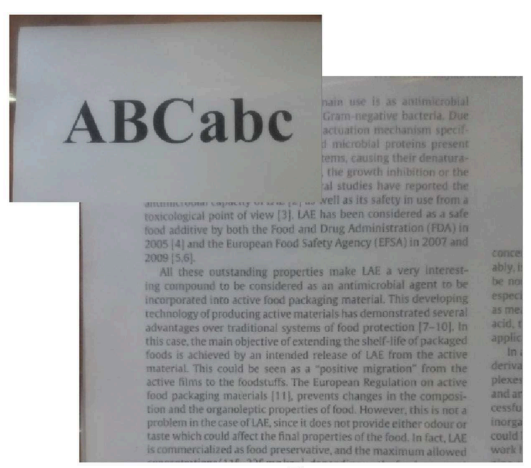

B

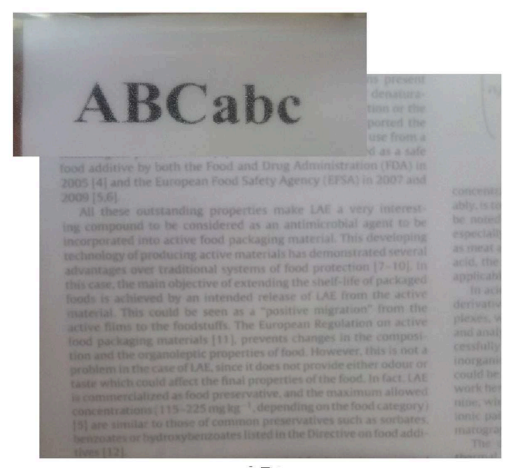

D

FIGURE 5 | Pictures of the SC coated films at different concentration of active agent: 0\% LAE (A), 5\% LAE (B), 10\% LAE (C), and 20\% LAE (D).

the increase in film cloudiness, with respect to the SC, SC5 and SC10 samples, is also visible in Figure 5.

\section{CONCLUSIONS}

In this work, innovative multifunctional and eco-sustainable antimicrobial packaging solutions have been successfully developed, through a conventional technique commonly applied in packaging industry. The produced films combine the structural and barrier performance of PET with the PLA sealing capacity and the LAE antimicrobial activity.

The chemical, physical and functional analyses carried out on the active multilayer films showed the good dispersion and homogeneous distribution of LAE into the polymer matrix, as well as the good adhesion of the coating layer on the substrate. Moreover, LAE addition did not modify the macromolecular structure of PLA.

Microbiological tests pointed out the effectiveness of the produced systems in releasing the antimicrobial agent and inhibiting microbial growth. In particular, the inhibition of microbial strain was found proportional to the LAE concentration into the PLA matrix, with $5.17 \log$ decrease of viable counts at 5\% LAE and total inhibition measured for coating formulations at higher LAE content.

In particular, it was pointed out that, by increasing the LAE concentration up to $10 \%$, the functional performance of the films, in terms of adhesion, water vapor barrier and optical properties, were not significantly affected. On the other hand, increasing LAE content at $20 \%$, a worsening of the PET film functionalities was observed. On the basis of these results, it comes out that the $10 \%$ LAE configuration allowed to obtain the best performance in terms of total microbial inhibition and functional properties.

\section{DATA AVAILABILITY STATEMENT}

All datasets generated for this study are included in the manuscript/supplementary files.

\section{AUTHOR CONTRIBUTIONS}

$\mathrm{AA}$ and LI: conceived and designed the experiments. AA: performed the experiments and the original draft preparation. AA, LD, and PS: conceptualization. LD, PS, and LI: review and editing of the manuscript. All authors discussed and revised the manuscript.

\section{ACKNOWLEDGMENTS}

The authors would like to gratefully thank Prof. Zvi Hayouka (The Hebrew University of Jerusalem) for valuable discussion, and Prof. Sacha Lucchini (University of Salerno) for his support with microbiological analyses. 


\section{REFERENCES}

Apicella, A., Adiletta, G., Di Matteo, M., and Incarnato, L. (2019). Valorization of olive industry waste products for development of new eco-sustainable, multilayer antioxidant packaging for food preservation. Chem. Eng. Trans. 75, 85-90. doi: 10.3303/CET1975015

Apicella, A., Scarfato, P., D’Arienzo, L., Garofalo, E., Di Maio, L., and Incarnato, L. (2018b). Antimicrobial biodegradable coatings based on LAE for food packaging applications. AIP Confer. Proc. 1981:020010. doi: 10.1063/1.5045872

Apicella, A., Scarfato, P., Di Maio, L., Garofalo, E., and Incarnato, L. (2018a). Evaluation of performance of PET packaging films based on different copolyester $\mathrm{O}_{2}$-scavengers. AIP Confer. Proc. 1981:020130. doi: $10.1063 / 1.5045992$

Attaran, S. A., Hassan, A., and Wahit, M. U. (2015). Materials for food packaging applications based on bio-based polymer nanocomposites. J. Thermoplast. Compos. Mater. 30, 143-173. doi: 10.1177/0892705715588801

Aznar, M., Gómez-Estaca, J., Vélez, D., Devesa, V., and Nerín, C. (2013). Migrants determination and bioaccessibility study of ethyl lauroyl arginate (LAE) from a LAE based antimicrobial food packaging material. Food Chem. Toxicol. 56, 363-370. doi: 10.1016/j.fct.2013.02.018

Bakal, G., and Diaz, A. (2005). The lowdown on lauric arginate: food antimicrobial hammers away at plasma membrane, disrupting a pathogen's metabolic process. Food Qual. 12, 54-61. Available online at: https://www. foodqualityandsafety.com/article/the-lowdown-on-lauric-arginate/

Barbaro, G., Galdi, M. R., Di Maio, L., and Incarnato, L. (2015). Effect of BOPET film surface treatments on adhesion performance of biodegradable coatings for packaging applications. Eur. Polym. J. 68, 80-89. doi: 10.1016/j.eurpolymj.2015.04.027

Becerril, R., Manso, S., Nerin, C., and Gómez-Lus, R. (2013). Antimicrobial activity of Lauroyl Arginate Ethyl (LAE), against selected food-borne bacteria. Food Control 32, 404-408. doi: 10.1016/j.foodcont.2013.01.003

Bugatti, V., Brachi, P., Viscusi, G., and Gorrasi, G. (2019). Valorization of tomato processing residues through the production of active bio-composites for packaging applications. Front. Mater. 6:34. doi: 10.3389/fmats.2019.00034

Bugnicourt, E., Schmid, M., Nerney, O. M., Wildner, J., Smykala, L., Lazzeri, A., et al. (2013). Processing and validation of whey-protein-coated films and laminates at semi-industrial scale as novel recyclable food packaging materials with excellent barrier properties. Adv. Mater. Sci. Eng. 2013, 1-10. doi: 10.1155/2013/496207

Chi-Zhang, Y., Yam, K., and Chikindas, M. L. (2004). Effective control of Listeria monocytogenes by combination of nisin formulated and slowly released into a broth system. Int. J. Food Microbiol. 90, 15-22. doi: 10.1016/S0168-1605(03)00168-5

Coronel-León, J., López, A., Espuny, M. J., Beltran, M. T., Molinos-Gómez, A., Rocabayera, X., et al. (2016). Assessment of antimicrobial activity of $\mathrm{N}^{\alpha}$-lauroyl arginate ethylester $\left(\mathrm{LAE}^{\circledR}\right)$ against Yersinia enterocolitica and Lactobacillus plantarum by flow cytometry and transmission electron microscopy. Food Control 63, 1-10. doi: 10.1016/j.foodcont.2015.10.050

Di Maio, L., Marra, F., Apicella, A., and Incarnato, L. (2017). Evaluation and modeling of scavenging performances of active multilayer PET based films for food preservation. Chem. Eng. Trans. 57, 1879-1884. doi: 10.3303/CET1757314

European Commission (2009). Commission regulation (EC) No 450/2009 of 29 May 2009 on active and intelligent materials and articles intended to come into contact with food. Off. J. Eur. Union L135, 3-11. Available online at: http://data. europa.eu/eli/reg/2009/450/oj

Gaikwad, K. K., Lee, S. M., Lee, J. S., and Lee, Y. S. (2017). Development of antimicrobial polyolefin films containing lauroyl arginate and their use in the packaging of strawberries. J. Food Meas. Charact. 11, 1706-1716. doi: 10.1007/s11694-017-9551-0

Haghighi, H., De Leo, R., Bedin, E., Pfeifer, F., Siesler, H. W., and Pulvirenti, A. (2019). Comparative analysis of blend and bilayer films based on chitosan and gelatin enriched with LAE (lauroyl arginate ethyl) with antimicrobial activity for food packaging applications. Food Pack. Shelf Life 19, 31-39. doi: 10.1016/j.fpsl.2018.11.015

Halász, K., Hosakun, Y., and Csóka, L. (2015). Reducing water vapor permeability of poly(lactic acid) film and bottle through layer-by-layer deposition of greenprocessed cellulose nanocrystals and chitosan. Int. J. Polym. Sci. 2015:954290. doi: $10.1155 / 2015 / 954290$
Higueras, L., Lopez-Carballo, G., Hernandez-Munoz, P., Gavara, R., and Rollini, M. (2013). Development of a novel antimicrobial film based on chitosan with LAE (Ethyl-N $\alpha$-dodecanoyl-1-arginate) and its application to fresh chicken. Int. J. Food Microbiol. 165, 339-345. doi: 10.1016/j.ijfoodmicro.2013.06.003

Infante, M., Pinazo, A., and Seguer, J. (1997). Non-conventional surfactants from amino acids and glycolipids: structure, preparation and properties. Colloids Surf. A 123-124, 49-70. doi: 10.1016/S0927-7757(96)03793-4

Lagaron, J. M. (2011). Multifunctional and Nanoreinforced Polymers for Food Packaging. Cambridge: Woodhead Publishing.

Landi, C., Paciello, L., De Alteriis, E., Brambilla, L., and Parascandola, P. (2014). High cell density culture with S. cerevisiae CEN.PK113-5D for IL-1 $\beta$ production: optimization, modeling, and physiological aspects. Bioproc. Biosyst. Eng. 38, 251-261. doi: 10.1007/s00449-014-1264-8

Li, G., Shankar, S., Rhim, J.-W., and Oh, B.-Y. (2015). Effects of preparation method on properties of poly(butylene adipate-co-terephthalate) films. Food Sci. Biotechnol. 24, 1679-1685. doi: 10.1007/s10068-015-0218-5

Lindner, M., Rodler, N., Jesdinszki, M., Schmid, M., and Sängerlaub, S. (2017). Surface energy of corona treated PP, PE and PET films, its alteration as function of storage time and the effect of various corona dosages on their bond strength after lamination. J. Appl. Polym. Sci. 135:45842. doi: 10.1002/app.45842

Molling, J., Seezink, J., Teunissen, B., Muijrers-Chen, I., and Borm, P. (2014). Comparative performance of a panel of commercially available antimicrobial nanocoatings in Europe. Nanotechnol. Sci. Appl. 7, 97-104. doi: 10.2147/NSA.S70782

Moreno, O., Gil, À., Atarés, L., and Chiralt, A. (2017). Active starch-gelatin films for shelf life extension of marinated salmon. LWT Food Sci. Technol. 84, 189-195. doi: 10.1016/j.lwt.2017.05.005

Muriel-Galet, V., López-Carballo, G., Gavara, R., and HernándezMuñoz, P. (2012). Antimicrobial food packaging film based on the release of LAE from EVOH. Int. J. Food Microbiol. 157, 239-244. doi: 10.1016/j.ijfoodmicro.2012.05.009

Muriel-Galet, V., López-Carballo, G., Hernández-Muñoz, P., and Gavara, R. (2014). Characterization of ethylene-vinyl alcohol copolymer containing lauril arginate (LAE) as material for active antimicrobial food packaging. Food Pack. Shelf Life 1, 10-18. doi: 10.1016/j.fpsl.2013.09.002

Nerin, C., Becerril, R., Manso, S., and Silva, F. (2016). "Ethyl Lauroyl Arginate (LAE): antimicrobial activity and applications in food systems," in Antimicrobial Food Packaging, ed J. Barros-Velázquez (London: Elsevier Science), 305-312.

Otero, V., Becerril, R., Santos, J. A., Rodríguez-Calleja, J. M., and Cristina Nerín, García-López, M. L. (2014). Evaluation of two antimicrobial packaging films against Escherichia coli O157:H7 strains in vitro and during storage of a Spanish ripened sheep cheese (Zamorano). Food Control 42, 296-302. doi: $10.1016 /$ j.foodcont.2014.02.022

Owens, D. K., and Wendt, R. C. (1969). Estimation of the surface free energy of polymers. J. Appl. Polym. Sci. 13, 1741-1747. doi: 10.1002/app.1969.070130815

Paciello, L., Falco, F. C., Landi, C., and Parascandola, P. (2013). Strengths and weaknesses in the determination of yeast cell viability by ATPbased bioluminescence assay. Enzyme Microb. Technol. 52, 157-116. doi: 10.1016/j.enzmictec.2012.12.011

Paciello, L., Landi, C., Orilio, P., Di Matteo, M., Zueco, J., and Parascandola, P. (2015). Bread making with Saccharomyces cerevisiae CEN.PK113-5D expressing lipase A from bacillus subtilis: leavening characterisation and aroma enhancement. J. Food Sci. Technol. 50, 2120-2128. doi: 10.1111/ijfs. 12876

Pezo, D., Navascués, B., Salafranca, J., and Nerín, C. (2012). Analytical procedure for the determination of Ethyl Lauroyl Arginate (LAE) to assess the kinetics and specific migration from a new antimicrobial active food packaging. Anal. Chim. Acta. 745, 92-98. doi: 10.1016/j.aca.2012.07.038

Robertson, G. L. (2013). Food Packaging Principles and Practice, $3 r d$ Edn. Boca Raton, FL: CRC Press.

Rubilar, J. F., Candia, D., Cobos, A., Díaz, O., and Pedreschi, F. (2016). Effect of nanoclay and Ethyl-N $\alpha$-dodecanoyl-L-arginate hydrochloride (LAE) on physico-mechanical properties of chitosan films. LWT Food Sci. Technol. 72, 206-214. doi: 10.1016/j.lwt.2016.04.057

Scarfato, P., Avallone, E., Galdi, M. R., Di Maio, L., and Incarnato, L. (2015a). Preparation, characterization, and oxygen scavenging capacity of biodegradable $\alpha$-tocopherol/PLA microparticles for active food packaging applications. Polym. Compos. 38, 981-986. doi: 10.1002/pc.23661 
Scarfato, P., Di Maio, L., and Incarnato, L. (2015b). Recent advances and migration issues in biodegradable polymers from renewable sources for food packaging. J. Appl. Polym. Sci. 132:42597. doi: 10.1002/app. 42597

Scarfato, P., Di Maio, L., Milana, M. R., Giamberardini, S., Denaro, M., and Incarnato, L. (2017). Performance properties, lactic acid specific migration and swelling by simulant of biodegradable poly(lactic acid)/nanoclay multilayer films for food packaging. Food Addit. Contam. Part A 34, 1730-1742. doi: 10.1080/19440049.2017.1321786

Wicochea-Rodríguez, J. D., Chalier, P., Ruiz, T., and Gastaldi, E. (2019). Active food packaging based on biopolymers and aroma compounds: how to design and control the release. Front. Chem. 7:398. doi: 10.3389/fchem.2019.00398

Zainab, W. A., and Dong, Y. (2019). Biodegradable and water resistant poly(vinyl) alcohol (PVA)/starch (ST)/glycerol (GL)/halloysite nanotube (HNT) nanocomposite films for sustainable food packaging. Front. Mat. 6:58. doi: $10.3389 /$ fmats. 2019.00058
Zonder, L., Mccarthy, S., Rios, F., Ophir, A., and Kenig, S. (2014). Viscosity ratio and interfacial tension as carbon nanotubes distributing factors in melt-mixed blends of polyamide 12 and high-density polyethylene. Adv. Polym. Technol. 33:21427. doi: 10.1002/adv.21427

Conflict of Interest: The authors declare that the research was conducted in the absence of any commercial or financial relationships that could be construed as a potential conflict of interest.

Copyright (c) 2019 Apicella, Scarfato, Di Maio and Incarnato. This is an open-access article distributed under the terms of the Creative Commons Attribution License (CC $B Y)$. The use, distribution or reproduction in other forums is permitted, provided the original author(s) and the copyright owner(s) are credited and that the original publication in this journal is cited, in accordance with accepted academic practice. No use, distribution or reproduction is permitted which does not comply with these terms. 